Journal of Computer Science 7 (1): 114-119, 2011

ISSN 1549-3636

(C) 2011 Science Publications

\title{
Mobile Element Scheduling for Efficient Data Collection in Wireless Sensor Networks: A Survey
}

\author{
K. Indra Gandhi and P. Narayanasamy \\ Department of Information Science and Technology, \\ Anna University, Chennai, 600025, India
}

\begin{abstract}
Problem statement: Wireless Sensor Networks (WSN) has become a very interesting field of research and has been deployed for various applications. The data sensed by the sensors need to be transmitted over to a Base Station (BS) where the data processing is done. Approach: The existing systems generally employ Multi-hop routing for data aggregation at the BS, where several nodes may forward data packets to the BS. This will lead to the reduced lifetime of the network and reduced battery life for sensor nodes near the BS, as they have to relay data from all parts of the network to the BS. Results: A simple yet efficient method to improve the network lifetime is designed with the help of Mobile Element (ME). The MEs act as mechanical carriers which move around in the sensing field, collecting the data from the sensors and transmitting them to the BS. In a relatively larger network, a single mobile element might not serve the purpose. So Rendezvous Points (RP) can be used in the network, to enhance the performance of the WSN. Conclusion/Recommendations: This article discusses the state of the art and major research challenges in scheduling these ME and the arising need for multiple MEs also being outlined.
\end{abstract}

Key words: Mobile element, wireless sensor networks, scheduling, data collection, base station

\section{INTRODUCTION}

\begin{tabular}{|c|c|c|c|}
\hline $\begin{array}{l}\text { A typical } \\
\text { onsists }\end{array}$ & $\begin{array}{l}\text { Wireless } \\
\text { of }\end{array}$ & $\begin{array}{c}\text { Sensor } \\
\text { spatially }\end{array}$ & $\begin{array}{r}\text { Network (WSN) } \\
\text { distributed }\end{array}$ \\
\hline
\end{tabular}
physical or environmental conditions, such as temperature, sound, vibration, pressure, motion, at different locations. The development of WSNs was motivated by military applications. However, WSNs are now used in civilian applications, including environment, traffic and habitat monitoring, healthcare and home automation.

The main challenges in the sensor networks are limited battery power and buffer overflow. An analysis of performance of the various existing methods for data collection in sensor networks has been performed. Implementation of all the basic ME Scheduling algorithms is performed to analyze their characteristics. Then, rendezvous-based system is implemented and its performance is determined. This study aims at analysing these algorithms for sensed data collection using an enhanced ME-based approach, which minimizes the losses in the system, improves the performance of the WSN, besides enhancing the lifetime of the network.
The multiple MEs approach in combination with the rendezvous system is analyzed. In the multiple MEs system, each ME to visit a certain set of RP based on the network topology and the number of RP obtained in the system.

The data sensed by the sensor nodes have to be transferred to the Base Station (BS) for further analysis by their respective field experts. Usually, in a multi hop network formed by the sensor nodes the readings are relayed to the BS for further processing. This leads to the frequent battery drain of the nodes near the BS. To avoid this, researchers have proposed (Xang et al., 2008) mobility as a solution for data gathering. The mobile nodes which act as mechanical carriers collect data from all the nodes and transfers to the BS. The problem of scheduling the mobile node such that none of the buffers overflow is termed as the Mobile Element Scheduling problem (Xang et al., 2008).

Several scheduling algorithms have been proposed in the literature (Xang et al., 2008; Somasundara et al., 2007; Gu et al., 2005; 2006; Ma and Yang, 2008; Gandhi et al., 2008; Pon et al., 2005; Slijepcevic and Potkonjak, 2001) which focuses on scheduling the mobile node such that there is no data loss. Although the aim of these scheduling algorithms is that there should be no loss of the sensed data, practical solutions 
will concentrate on minimizing the data loss. This is because, when there is an increase in the number of sensor nodes, obviously the data loss will increase. Our major concern will be to analyze the impact of the scheduling algorithms under different scenarios. Also another important concept of topology management (Godfrey and Ratajczak, 2004) is to have only a subset of nodes actively participating in the network, thus creating less communication and conserving energy in nodes.

This study presents a survey of the existing scheduling algorithms for the Mobile Element (ME) in Wireless Sensor Networks (WSN).

Figure1 illustrates the scheduling algorithms for the ME and based upon the analysis, the problem has been extended to multiple ME scheduling problem. The Earliest Deadline First (EDF) exhibits the characteristics of scheduling the ME starting with the least deadline nodes. EDF with K-look ahead schedules with reference to the $\mathrm{k}$ value. The schedules are formulated with reference to the look-up of $\mathrm{k}$ nodes. The Minimum Weighted Sum First (MWSF) schedules are done based upon a weight calculated on the deadline of the sensor nodes. In the scheduling process, the ME's visit is scheduled only for these boundarynear nodes as the aggregated data is available in this boundary-near nodes. Rendezvous Points finds the optimal RPs when MEs move along the data routing tree in order to perform data collection.

A set of source nodes periodically sense and accumulate data that must be delivered to the BS before their deadlines expire. Relaying mechanisms using multi-hop routing are not efficient as they result in a lot of resource wastage in the network and the battery life of the sensor nodes deteriorate rapidly if they relay their data to other nodes. So, we use MEs, to collect the data from the sources and deposit them at the BS. To avoid the deadline expiration in a larger network and to reduce the overhead on the ME, a subset of nodes referred to as RP which buffer the data obtained from the nodes, from where the MEs can pick up the data was a proposed solution. By using multiple MEs the delay can be further reduced and enhance the performance of the network. Reduction of data loss is the objective of the scheduling algorithms keeping into account the individual node's capability of sensing, forwarding, aggregating and relaying the data to the BS.

\section{Factors influencing mobile:}

Element scheduling:

Mobile elements: Mobile Elements can be deliberately built into the system to improve the lifetime of the network and act as mechanical carriers of data, which move around in the sensing field. The mobile element, whose mobility is controlled, visits the nodes to collect their data before their buffers are full.

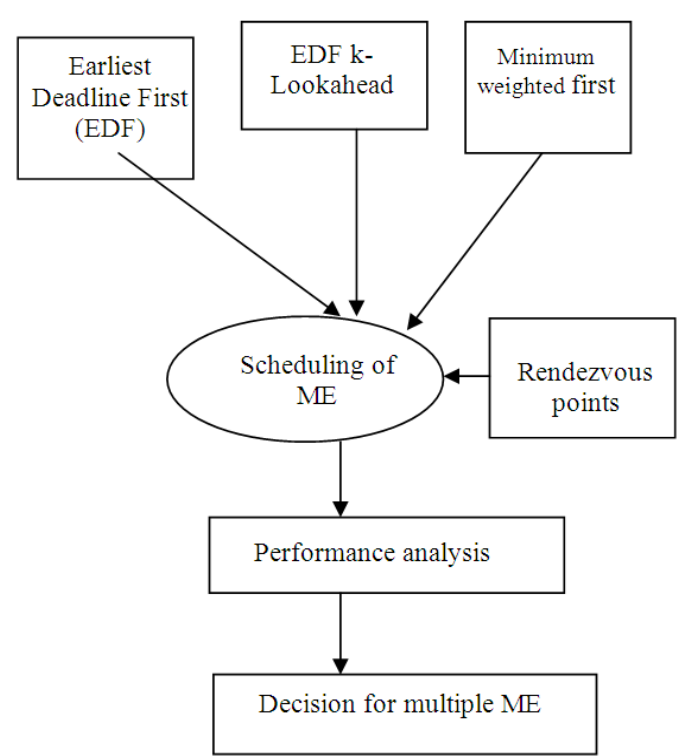

Fig. 1: State of the art scheduling algorithms

Buffer size: The sensors sense data from the surroundings and accumulate data over a period of time. These data need to be transmitted to the BS and again samples of data are sensed by the sensors periodically. The buffer at the sensors should be periodically cleared, or else, the data in the buffer gets replaced by newer data. The buffer size at the sensor nodes needs to be optimal enough to minimize the data losses in the system.

Battery life: The major reason for the introduction of the MEs in the system is to improve the battery life of the sensor nodes. When data are relayed to BS through multiple hops, all the nodes must be active forever and hold the details of the complete routing tree. The nodes near the BS will be relaying data to BS from all parts of the network, so their battery drains rapidly and hence, will fail.

Sensor node deadlines: The deadline value for each sensor node is determined from its data sampling rate. When the ME doesn't fetch the data from the sensor node before the deadline, data loss occurs. The node's deadlines can be homogeneous or heterogeneous, based on the requirements of the network. The scheduling of the MEs is primarily done based upon the set of deadline values, to minimize the losses and to cover the maximum path possible in the wireless sensor network.

Rendezvous Points (RPs): The RPs are a subset of nodes from which the MEs can pick up the data, instead 
of traversing all the nodes. The source nodes sense the data and relay it to its nearest RP in the network. RPs accumulate the data of more than a single source node until the ME arrives to fetch that data. So, the buffer size at the RP is an important issue, as they need to buffer all the constituent data till the ME arrives.

Multiple mobile elements: In very large networks, a single ME will not be sufficient to prevent the deadline expiration problem. In this case, using multiple MEs can solve the problem. Each MEs is allocated a set of Sensor Nodes. The ME collects data only from allocated set of sensor nodes.

State of the art: Mobile element scheduling algorithms: In WSN, the real-time data sensed by the nodes are to be sent to the BS periodically, as they keep varying from time to time and stale data need to be eliminated. In this scenario, the relaying and forwarding mechanisms were initially used to transmit data to the base station. As this was found to be highly disadvantageous, the concept of using MEs in the sensor network has been proposed by researchers. These MEs perform the functionality of data collection in the network.

Godfrey and Ratajczak (2004), the implementation of MEs for data collection in a wireless sensor network is discussed. To avoid the draining of battery life, some mobile nodes, known as the MEs are built into the system. They act as mechanical carriers which move around in the sensing field, collect the data from the sensor nodes and transmit them to the base station.

Somasundara et al. (2007), the various techniques of scheduling a single ME to visit all the sensor nodes in wireless sensor network are discussed. The ME, whose mobility is controlled, visits the nodes to collect their data before their buffers are full. In general, the spatio-temporal dynamics of the sensed phenomenon may require sensor nodes to collect samples at different rates, in which case, some nodes need to be visited more frequently than others. This work formulates the problem of scheduling the ME in the network so that there is no data loss due to buffer overflow. If we use a single ME to collect the data from the sensor nodes, the order in which the ME visits to the nodes is scheduled mainly based on the deadlines of the nodes, upon the expiration of which, the buffers overflow. An example of a basic scheduling algorithm used for scheduling the $\mathrm{ME}$ is the EDF algorithm and its extensions. Some other algorithm implementations are also discussed in this work, which will further reduce the data losses in the system.
Xang et al. (2008), a new approach called the RPs approach is discussed. The ME was initially considered to traverse all the nodes in the network based on primitive scheduling algorithms and collect the data from the source nodes. A rendezvous-based approach is used for making the ME more efficient. A subset of nodes serves as the Rendezvous Points (RPs) in the network. They buffer data originated from the data sources. They transfer the buffered data to the ME, thus enabling the ME to collect large volume of data without having to travel long distances. The data, which originate at sources, are relayed to the RPs almost immediately. Hence the need for scheduling the individual nodes considering their individual deadlines does not arise. Instead, the RPs are traversed by the MEs.A set of nodes referred to as RPs are determined from which the MEs can pick up the data originated from sources and transport to the BS before the deadlines.

Data packets in the wireless network should be sent to a fixed base station, where the data from various nodes are collected. Packets need to be transmitted over the wireless network through other nodes. This process is called Relaying. The intermediate nodes need to forward the packets towards the destination nodes. The sensor nodes near the base station are highly operative almost all the time as they should relay the data to the BS from various parts of the network. This leads to a non-uniform depletion of resources in those nodes and the battery life drains rapidly. If the BS is distant, with the increasing number of hop-counts, the propagation time for the data transmission is very high. Each sensor node is assigned a buffer for accumulating the sensed data.

To overcome this problem of deterioration of battery life and to improve the network lifetime, MEs were implementation of these mechanical carriers realized through a mobile node which is also termed as the ME to collect the data according to it's own capability of storing the aggregated data.

The issue with the ME is that the order in which it traverses through the nodes of a network should ensure minimal data losses. Some of the major algorithms used to schedule the ME are Earliest Deadline First (EDF), EDF with k-look ahead, Minimum Weighted Sum First algorithms.

The EDF algorithm is the simplest of all ME Scheduling algorithms. In this algorithm, the node which has the least deadline value is visited first, irrespective of all other constraints. The advantage of this is that, it is very easy to be implemented in a sensor network and less complexity is involved. But one obvious shortcoming of this algorithm is that it does not take into account the cost 
values and relies only on deadlines. i.e., a ME, instead of considering an optimal path, will try to reach a very farther node with a smaller deadline. But because of the higher cost involved, this leads to several failed nodes which could have been avoided.

They act as mechanical carriers of data, which move around in the sensing field. The ME, whose mobility is controlled, visits the other nodes to collect their data before their buffers are full.

MEs are nodes whose only job is to collect data from these sensing nodes and to buffer it to the BS. The EDF-k look ahead algorithm is an extension of EDF algorithm. In this, the ME does not visit the node where the deadline expires immediately, instead the deadlines of the next $\mathrm{k}$ nodes is analyzed, to know if all the nodes satisfy their deadlines. Only if all the deadlines are satisfied, then the ME goes to the first node in the selected path. It is important to note that scheduling $\mathrm{k}$ visits at a time is not done, but, instead, for each visit, $\mathrm{k}$ nodes are looked at and the next node is chosen. The look ahead algorithm takes care of nodes with same deadline values, whereas EDF would have chosen randomly depending on in what order they appeared in the sorted array.

The Minimum Weighted Sum First algorithm is different from the normal EDF algorithm because in addition to considering the deadlines, it also takes into account the cost of each edge in the network. $\alpha$ value involved with this is chosen based on the network topology, where it can be advantageous to reduce losses. When $\alpha<0.5$, the scheduling is performed predominantly based on the cost. When $\alpha>0.5$, it relies more on the deadlines. With a single ME, Minimum Weighted Sum First is found to perform better than EDF with $\alpha<=0.1$.

In a large network, it might not be efficient for a single ME to traverse all nodes of the network. Instead, a subset of nodes, called as RPs, buffer the data originating at the source nodes, for MEs to fetch from those points. This will reduce the cost overhead on the ME.

The algorithms mentioned above are just a way to schedule the ME visits to various parts of the network for data collection. The battery life, which is one of the key constraints for switching over to the mobileelement based approach, improves considerably when compared with the multi-hop networks. And the data losses are also found to reduce progressively with each approach. Each ME is assigned to visit a set of RPs in the system to collect data from them. This further reduces data loss in the system.

\section{MATERIALS AND METHODS}

The implementation of the various algorithms is carried out in OMNeT++ simulator and the performance characteristics of all the methods are analyzed. In these implementations, the system is executed for different scenarios - varying costs, varying deadlines, etc. These different scenarios are studied together.

Mobile element schedule with multiple mobiles: We can see that by assigning single mobile element to the network lot of nodes reach the deadline due to buffer overflow. To reduce it we go for rendezvous points assigning to the nodes in the WSN. The sensor nodes are made to relay the data to the nearest rendezvous points using multi hop relying mechanism and the mobile node collect the data gathered in it. By doing so the network lifetime is increased by reducing the no. of nodes meeting deadline.

This mechanism can be extended by using multiple mobile elements in which each mobile element is assigned a set of rendezvous points and the mobile elements collect the data from these RPs.

The number of mobile elements can be varied for a given network. And these mobile elements are assigned a set of RPs in Round-Robin fashion. The mobile elements visit only those set of RPs and gather data collected from them. By this mechanism, we can reduce the nodes meeting deadline to a great extent. By increasing the number of mobile elements in the network the number of node failure can be further reduced.

\section{RESULTS AND DISCUSSION}

The existing algorithms EDF, EDF-K and MWSF have been simulated for varying the cost in the sense the nodes have been deployed randomly and the distance to travel each node will differ.

As shown in Fig. 2, the variation in cost factor shows the node failure to be more for EDF compared to the other two algorithms.

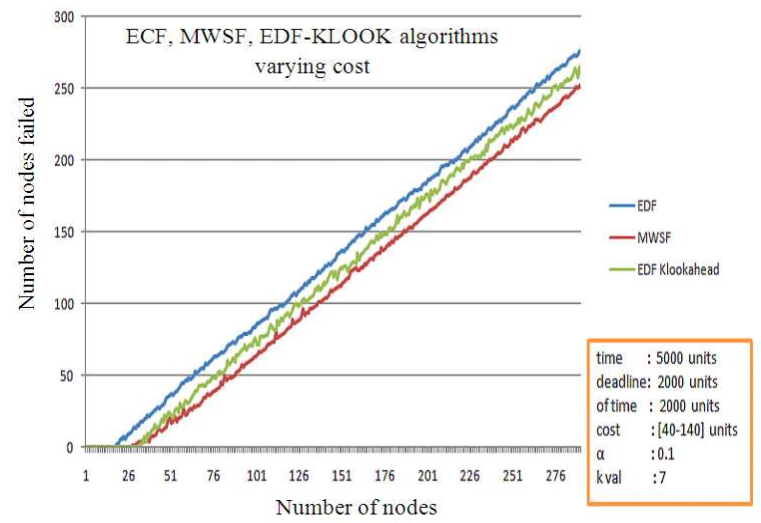

Fig 2 Impact of cost variation 

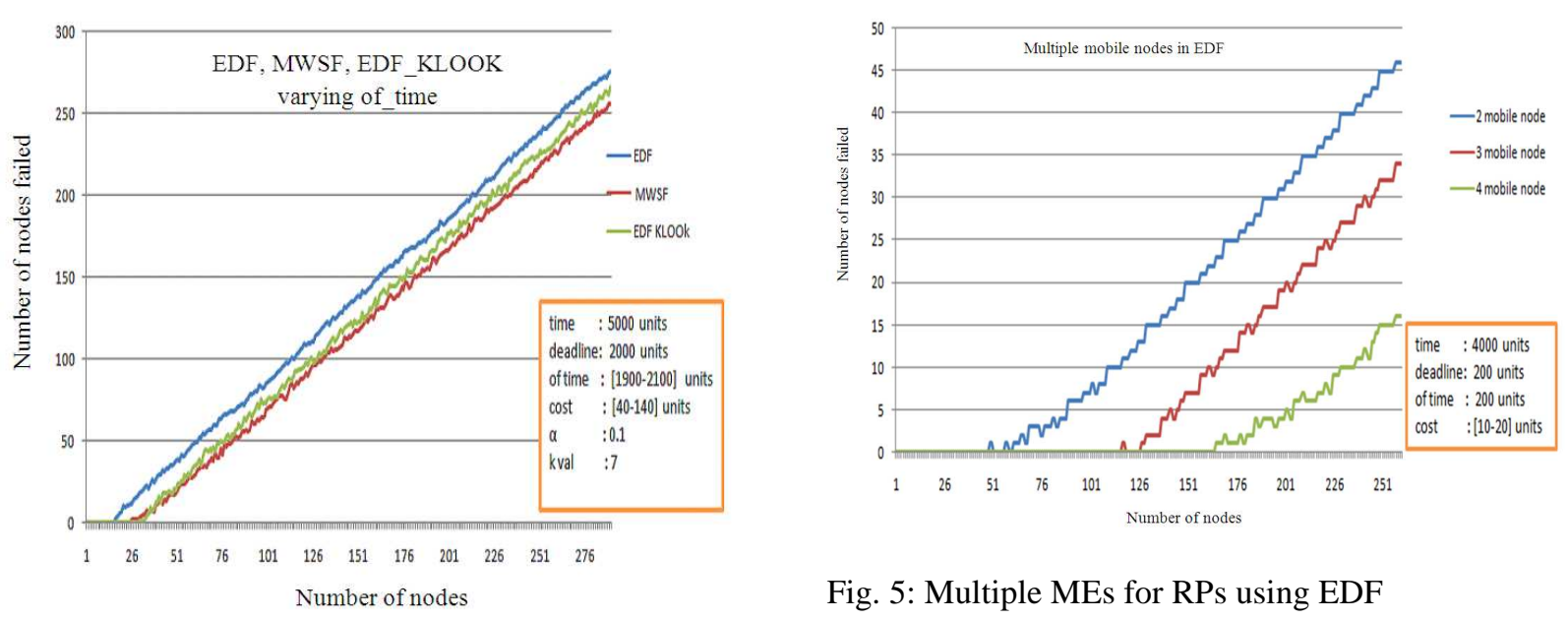

Fig. 3: Varying overflow time (of-time)

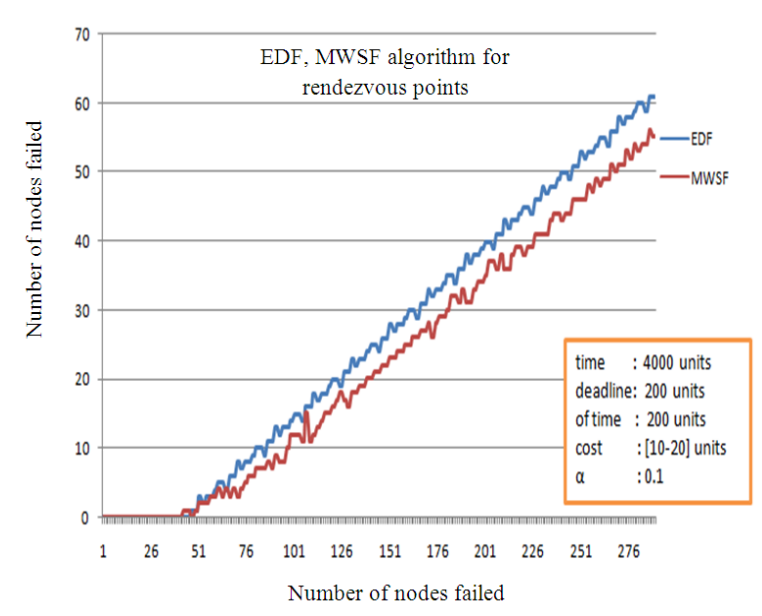

Fig. 4: Behavior of the scheduling algorithms using Rendezvous Points (RPs)

From Fig. 3, it is evident that the variation of the overflow time (the nodes sense the data at different sampling rate) also implies that the EDF algorithm proves to have more number of nodes missing their deadlines.

In Fig. 4, after identifying the RPs, EDF and MWSF is applied to these RPs which will obviously reduce the number of nodes failed i.e., the M.E misse to visit the nodes before their deadlines. EDF-k lookahead is not taken into consideration since the determination of RPs will lead to a minimum subset of nodes. This minimum set of nodes will converge the EDF algorithm into EDF-k lookahead algorithm and hence only EDF and MWSF algorithms are taken into consideration. Thus from Fig. 4, it is clear that upto 50 nodes there is no node failure.

Fig. 5: Multiple MEs for RPs using EDF

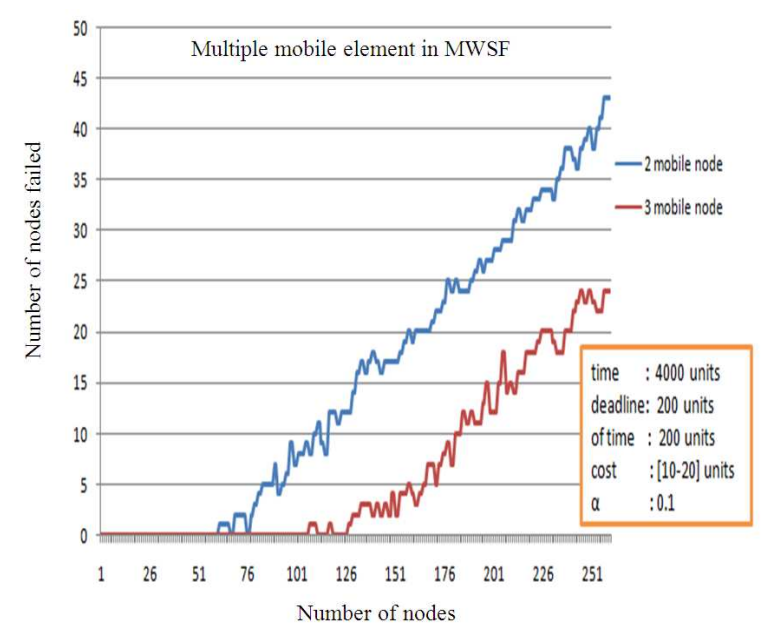

Fig. 6: Multiple MEs for RPs using MWSF performs better as the no. of ME increases, node failure i.e., the data collection has been more compared to single ME.

As the nodes grow, the misses also grow but with the fact that the misses now shrinks to 50 units when compared to executing the same algorithm without RPs. One obvious point to note is that the node failure as illustrated in Fig. 2-6 is the ME which failed to collect the data from the nodes which had stored the sensed data.

Using RPs the algorithms perform better with the consideration that the nodes acting as RPs should be with additional capabilities such as power, storage buffer of these nodes should be more compared to the other nodes. In Fig.6, the EDF algorithm was again analyzed in terms of multiple MEs and it was found that as the MEs are increased, the number of nodes that failed to store their data before the visit of the ME have been decreasing. In Fig. 6, the MWSF algorithm was simulated with 2 and 3 mobile nodes and it was found 
that with EDF, when the number of MEs is 4, the node failed to dispatch there is 25 . The same observation can be made for multiple MEs with MWSF in which the same result can be observed when the number of MEs is 3 .

From the existing algorithms, it can be identified that the RPs with MWSF gives a better performance when the number of MEs is determined to be 3. The RPs identified should have additional capabilities compared to the other sensor nodes which is a challenging task. The power consumption of the RPS will be more since they act as collection points which can be considered for future study.

\section{CONCLUSION}

From the experimentation results obtained, it has been found that this approach using the RPs in combination with the multiple MEs is found to be advantageous over the existing systems of data collection in a large scale WSN. After running the simulation over a number of trials by varying the parameters like the number of sensor nodes, costs, deadlines, etc. it is found that the performance of the system is greatly improved by implementing the MWSF algorithm for RPs using multiple Mobile Elements.

In the future, this work can be extended to heterogeneous WSNs by taking advantage of the nodes with more computational power and storage capacity. The current formulation does not include the energy expenditure for movement. However, energy can be easily modeled as a function of distance moved. In addition, the speed of the mobile element may be made variable, which may result in added benefits.

\section{REFERENCES}

Gandhi, K.I., D. Rajasekar and M.M.P. Shyam, 2008. A cluster-based hierarchical approach for scheduling the mobile element in wireless sensor networks. Proceeding of the International Conference on High Performance, (ICHP'08), Anna University, India, pp: 1-5.
Godfrey, P. and D. Ratajczak, 2004. Naps: scalable, robust topology management in wireless ad hoc networks. Proceeding of the 3rd International Symposium Information Processing in Sensor Networks, Apr. 26-27, California University, USA., $\quad$ pp: 443-451. DOI: 10.1109/IPSN.2004.1307366

Gu, Y., D. Bozdag, E. Ekici, F. Ozguner and R.W. Brewer, 2005. Partitioning based mobile element scheduling in wireless sensor networks. Proceeding of the IEEE Sensor Conference, Sep. 26-29, Ad Hoc Communication and Networks, USA., pp: 386-395. DOI: 10.1109/SAHCN.2005.1557092

Gu, Y., D. Bozdag, R.W. Brewer and E. Ekici, 2006. Data harvesting with mobile elements in wireless sensor networks. Comput. Networks, 50: 3449-3465.

Ma, M. and Y. Yang, 2008. Data gathering in wireless sensor networks with mobile collectors. Proceeding of the IEEE International Symposium, Apr. 14-18, Parallel and Distributed Processing, New York, pp: 1-9. DOI: 10.1109/IPDPS.2008.4536269

Pon, R., A. Batalin, J. Gordon, A. Kansal and D. Liu et al., 2005. Networked infomechanical systems: A mobile embedded networked sensor platform. Proceeding of the 4th International Conference Information Processing in Sensor Networks, Apr. 15, California University, USA., pp: 376-381. DOI: 10.1109/IPSN.2005.1440952

Slijepcevic, S. and M. Potkonjak, 2001. Power efficient organization of wireless sensor networks. Proceeding of the IEEE International Conference Communication, Jun. 11-14, California University, USA., $\quad$ pp: 472-476. DOI: 10.1109/ICC.2001.936985

Somasundara, A.A., A. Ramamoorthy and M.B. Srivastava, 2007. Mobile element scheduling with dynamic deadlines. IEEE Trans. Mobile Comput., 6: 296-305.

Xang, G., T. Wang, Z. Zie and W. Jia, 2008. Rendezvous planning in wireless sensor networks with mobile elements. IEEE Trans. Mobile Comput., 7: 1430-1443. 